\title{
Prevalence, genotyping and macrolide resistance of Mycoplasma pneumoniae among isolates of patients with respiratory tract infections, Central Slovenia, 2006 to 2014
}

R Kogoj ${ }^{1}$, T Mrvic $^{2}$, M Praprotnik ${ }^{3}$, D Kese ${ }^{1}$

1. University of Ljubljana, Medical Faculty, Institute of Microbiology and Immunology, Ljubljana, Slovenia

2. University Medical Centre Ljubljana, Department of Infectious Diseases, Ljubljana, Slovenia

3. University Medical Centre Ljubljana, Division of Paediatrics, University Children's Hospital, Ljubljana, Slovenia

Correspondence: Darja Kese (darja.kese@mf.uni-lj.si)

Citation style for this article:

Kogoj R, Mrvic T, Praprotnik M, Kese D. Prevalence, genotyping and macrolide resistance of Mycoplasma pneumoniae among isolates of patients with respiratory tract infections, Central Slovenia, 2006 to 2014. Euro Surveill. 2015;20(37):pii=30018. DOI: http://dx.doi.org/10.2807/1560-7917. ES.2015.20.37.30018

In this retrospective study we employed real-time polymerase chain reaction (PCR) to analyse the occurrence of Mycoplasma pneumoniae among upper and lower respiratory tract infections (RTI) in the Central Region of Slovenia between January 2006 and December 2014. We also used a culture and pyrosequencing approach to genotype strains and infer their potential macrolide resistance. Of a total 9,431 tested samples from in- and out-patient with RTI, 1,255 (13\%) were found to be positive by M. pneumoniae PCR. The proportion of positive samples was $19 \%(947 / 5,092)$ among children ( $\leq 16$ years-old) and $7 \%(308 / 4,339)$ among adults (>16 years-old). Overall, among those PCR tested, the highest proportions of M. pneumoniae infections during the study period were observed in 2010 and 2014 . In these two years, $18 \%(218 / 1,237)$ and $25 \%(721 / 2,844)$ of samples were positive respectively, indicating epidemic periods. From the 1,255 M. pneumoniae PCR-positive samples, 783 (614 from paediatric and 169 from adult patients) were successfully cultured. Of these, $40 \%(312 / 783)$ were constituted of strains belonging to the $\mathrm{P}_{1}$ type II genomic group, while $60 \%(469 / 783)$ contained strains of the $\mathrm{P}_{1}$ type I group. Two isolates comprised both $\mathrm{P}_{1}$ type I and II strains. Results of a genotype analysis by year, showed that the dominant $M$. pneumoniae $\mathrm{P}_{1}$ type during the 2010 epidemic was $P_{1}$ type II ( $82 \%$ of isolates; 81/99), which was replaced by $P_{1}$ type I in the 2014 epidemic $(75 \% ; 384 / 510)$. This observation could indicate that the two epidemics may have been driven by a type shift phenomenon, although both types remained present in the studied population during the assessed period of time. Only $1 \%$ of strains (7/783) were found to harbour an A2063G mutation in the 23S rRNA gene, which confers macrolide resistance, suggesting that the occurrence of $M$. pneumoniae macrolide resistance still seems to be sporadic in our geographic area.

\section{Introduction}

Mycoplasma pneumoniae is a fastidious, slow growing, cell wall-lacking bacteria that can be the causative agent of up to $40 \%$ cases of community-acquired respiratory tract infections (RTI) [1-6]. Although most cases present with mild or moderate symptoms, serious disease requiring hospitalisation occasionally occurs [7].

M. pneumoniae incidence usually increases during epidemics, which occur at intervals of four to seven years [8-10]. This pattern is presumably linked to the alternation of $\mathrm{P}_{1}$ adhesin types, which tend to change dominance over the course of time and, consequently, allow M. pneumoniae to elude the human host immune response [11]. According to nucleotide (nt) differences in two repetitive regions (RepMP2/3 and RepMP4) in the MPN141 gene, which codes for $P_{1}$ adhesin, $M$. pneumoniae strains can be divided into two genomic groups, called $\mathrm{P}_{1}$ type I and $\mathrm{P}_{1}$ type II [12], with several subtypes [13].

Macrolide-resistant strains among $M$. pneumoniae isolates are increasingly described, with reported resistance rates among studies from European Union Member States [11,14-17], Israel [18] and the United States [19] of up to $30 \%$. In contrast, rates of over $90 \%$ have been observed in some parts of Asia [20-25]. Low to high grade resistance against many if not all macrolides in $M$. pneumoniae has been shown to be related to $n t$ substitutions in two regions of the $\mathrm{V}$ domain in the $23 \mathrm{~S}$ rRNA gene, namely at positions 2,063, 2,064, 2,067 and 2,617 (M. pneumoniae numbering). Among 
Samples of patients with upper and lower respiratory tract infections tested by Mycoplasma pneumoniae real-time polymerase chain reaction and annual positivity rate, Central Slovenian region, 2006-2014 ( $\mathrm{n}=9,431$ samples)

\begin{tabular}{|c|c|c|c|c|c|c|c|c|c|c|}
\hline \multirow{2}{*}{ Samples } & \multicolumn{9}{|c|}{ Year } & \multirow{2}{*}{$\begin{array}{c}\text { Period } \\
2006-2014\end{array}$} \\
\hline & 2006 & 2007 & 2008 & 2009 & 2010 & 2011 & 2012 & 2013 & 2014 & \\
\hline $\begin{array}{l}\text { Total tested } \\
\mathrm{N}\end{array}$ & 391 & 470 & 393 & 659 & 1,237 & 1,185 & 1,005 & 1,247 & 2,844 & 9,431 \\
\hline $\begin{array}{l}\text { Positive } \\
\text { N (\%) }\end{array}$ & $9(2)$ & $19(4)$ & $16(4)$ & $48(7)$ & 218 (18) & 117 (10) & $46(5)$ & $61(5)$ & $721(25)$ & 1,255 (13) \\
\hline
\end{tabular}

these mutations, the A2063G mutation appears to be the most common, followed by A2064G [26]. All other mutations A2063C [27], A2063T [28], A2067G, C2617G and $C_{2617 A}$ are found much less frequently [26].

In Slovenia, M. pneumoniae infections are not notifiable and $M$. pneumoniae genotyping as well as antibiotic resistance testing are not yet provided as a routine service. Moreover, limited data are so far available for longitudinal studies looking into changes in the prevalence of $M$. pneumoniae infections, genetic diversity and antibiotic resistance.

In the only two published serology-based studies available from Slovenia, one from April 1996 to March 1997 [29] and the other from November 1999 to April 2001 [30], M. pneumoniae was frequently found as the causative agent of community-acquired pneumonia in hospitalised patients (5.7\% [29] and 24.8\% [30]). Based on these reports and the observation of an increased number of $M$. pneumoniae cases from routine laboratory testing all over Slovenia in 2010 and 2014 (data not shown), we decided to analyse the occurrence of M. pneumoniae infections in in- and out-patients with RTIs. At the end of 2005 , real-time polymerase chain reaction ( $P C R$ ) was introduced as the main diagnostic tool for $M$. pneumoniae infections in the Institute of Microbiology and Immunology at the University of Ljubljana, which covers the entire Central Slovenian Region. This allowed us to analyse tested samples from January 2006 to December 2014. We also used culture and molecular assays to further characterise the genotype of isolated strains during this period, and infer their potential macrolide resistance.

\section{Methods}

\section{Patient samples and Mycoplasma pneumoniae testing}

Our study was conducted between January 2006 and December 2014. Patients considered, were inhabitants of the Central Region of Slovenia, which on average represents $41 \%(843,528 / 2,038,281)$ [31] of the whole Slovenian population during the designated period of time. The distribution of patients did not have any apparent specific clustering in space and time. Thirtytwo per cent $(2,996 / 9,431)$ of patients were from the capital Ljubljana and $68 \%(6,435 / 9,431)$ from other parts of the Central Slovenian region. Depending on the severity of the symptoms, patients were treated as in- or out-patients. During the study period, all consecutive $(n=9,431)$ upper or lower respiratory tract specimens obtained from 5,092 (54\%) paediatric $(\leq 16$ years of age) and 4,339 (46\%) adult ( $>16$ years of age) patients with RTI were enrolled. Together with each sample, we received data of the patient's sex, age, address, attending physician and his/her institution, sample type, date and place of collection and basic diagnosis (RTI). An aliquot of each sample was subjected to routine laboratory testing by $M$. pneumoniae real-time PCR (Argene biosoft, France). This protocol was not changed during the time of the study. The remainder of each PCR positive sample was cultivated in order to obtain pure M. pneumoniae isolates.

\section{Culture of Mycoplasma pneumoniae positive samples}

Culture was performed by using Mycoplasma selective broth and agar plates (OXOID, United Kingdom (UK)) enriched with Mycoplasma supplement G or P (OXOID, UK) according to standard methods described elsewhere [32]. The obtained isolates were stored at $-80^{\circ} \mathrm{C}$ until further testing.

\section{Mycoplasma pneumoniae genotyping and} macrolide resistance detection

Neither mutations in the $23 \mathrm{~S}$ rRNA gene domain II, ribosomal proteins L4 and L22 [26], nor genomic or plasmid erm genes have been shown to be implicated in macrolide resistance in $M$. pneumoniae [33]. Moreover as M. pneumoniae harbours only one copy of the $23 \mathrm{~S}$ rRNA gene [34], molecular tests targeting the 23S rRNA gene domain $\mathrm{V}$ offer valuable tools for quick identification of resistant strains. Such tests have been developed, as well as some allowing rapid and reliable genotyping, and these can even be applied directly to clinical samples $[11,15,35-39]$. Recent publications by Spuesens et al. $[40,41]$ describe a pyrosequencing approach for genotyping $M$. pneumoniae and determination of macrolide resistance. Based on single nt polymorphisms (SNPs) in the MPN141 gene's constant region, and on additional information from the constant region of the MPN528a gene, the method enables quick classification of strains into $\mathrm{P}_{1}$ type I and $\mathrm{P}_{1}$ type II. Macrolide resistance implicated mutations in the two regions of the $23 \mathrm{~S}$ rRNA gene $\mathrm{V}$ domain can also be detected. This 
pyrosequencing approach was used for our study with some modifications which are further described.

Mycoplasma pneumoniae DNA was purified from 200 $\mu \mathrm{L}$ of liquid culture (OXOID, UK) using the MagNA Pure Compact automated DNA extraction system (Roche, Germany). A total of $5 \mu \mathrm{L}$ of the $200 \mu \mathrm{L}$ eluate was used in all $P C R$ reactions.

All primers, reaction conditions and master mix compositions used for the amplification of $M$. pneumoniae MPN141, MPN528a and two parts of the $23 \mathrm{~S}$ rRNA domain $\mathrm{V}$ (designated as Assay 1 - region encompassing macrolide resistance important positions 2,063, 2,064, 2,067 and Assay 2 - region encompassing macrolide resistance important position 2,617) were as described by Spuesens et al. [40], with several modifications. Briefly, for all PCR assays the number of cycles was increased to 50 , with the denaturation step prolonged to $15 \mathrm{~s}$ and the extension step shortened to $15 \mathrm{~s}$. The annealing step was $30 \mathrm{~s}$ with the temperature set to $55^{\circ} \mathrm{C}$ for all PCR assays. The master mix was as previously published [40]. The resulting biotinylated amplification products were checked for expected sizes and suitable amounts by $1.5 \%$ agarose gel electrophoresis stained with 1 X SYBR Safe (Invitrogen, Germany).

All remaining amplified fragments of MPN141, MPN528a, $23 S$ rRNA Assay 1 and Assay 2 were pyrosequenced on a PyroMarkID instrument (Biotage, Sweden) with PyroMark Gold Q96 SQA Reagents (Qiagen, Germany) using previously designed sequencing primers (TIB MOLBIOL, Germany) [40]. For the detection of $\mathrm{P}_{1}$ type specific SNP, we adopted the dispensation order developed earlier [40], but we constructed our own specific nt dispensation orders that cover all so far detected mutations linked with macrolide resistance in respective portions of the $M$. pneumoniae $23 \mathrm{~S}$ rRNA gene domain V. Moreover, the dispensation order for the $M$. pneumoniae $23 \mathrm{~S}$ rRNA pyrosequencing Assay 1 was extended to produce 79 bases long pyrosequencing products, which were later used to additionally check the specificity of the PCR assay, by National Center for Biotechnology Information (NCBI) basic local alignment tool (BLAST) search.

Genotype and possible macrolide resistance designations were attributed using IdentyFire software (Biotage, Sweden) loaded with custom built libraries.

\section{Mycoplasma pneumoniae 23S rRNA}

polymerase chain reaction Assay 1 and Assay 2 specificity

NCBI BLAST search of the primers [40] used in the $23 \mathrm{~S}$ rRNA domain V Assay 1 and Assay 2 showed a possible nonspecific annealing with Mycoplasma genitalium. Although $M$. genitalium is not a commonly recognised pneumonia agent and despite the fact that RTI isolates were used as starting material, we decided to additionally check the specificity of the primers. Both $M$. pneumoniae $23 \mathrm{~S}$ rRNA assays were therefore performed with DNA from Chlamydia pneumoniae, C. psittaci, C. trachomatis, Corynebacterium spp., Enterococcus fecalis, Escherichia coli, Klebsiella pneumoniae, Moraxella cattarhalis, Mycoplasma genitalium, Mycoplasma hominis, Neisseria gonorrhoeae, Proteus mirabilis, Pseudomonas aeruginosa, Staphylococcus aureus, Streptococcus pyogenes, Streptococcus agalactiae and Ureaplasma spp.

\section{Results}

\section{Patient samples and Mycoplasma pneumoniae testing}

From 9,431 patient samples, 1,255 (13\%) were found to be $M$. pneumoniae positive by PCR. Of the latter, $51 \%(n=640)$ were from males and $49 \%(n=615)$ from females. The median age of patients infected by $M$. pneumoniae was 8 years (range: 43 days to 85 years).

While M. pneumoniae was found among $19 \%$ $(947 / 5,092)$ of children samples, $7 \%(308 / 4,339)$ of adult samples tested positive. The highest proportion of positive samples were found in the age group comprising individuals between six and 16 years-old (26\%; $565 / 2,162)$, followed by preschool children $\leq 5$ years-old (13\%; 382/2,930), adults between 17 and 65 years-old (8\%; 288/3,253) and elderly persons $>65$ years-old $(2 \% ; 20 / 1,086)$.

A closer look at the distribution over time of positive patient samples revealed that the majority were found in 2010, 2011 and 2014, when 218/1,237 (18\%), $117 / 1,185(10 \%)$ and $721 / 2,844(25 \%)$ of patient samples contained detectable amounts of $M$. pneumoniae DNA, respectively (Table 1 ).

An even more detailed month to month analysis shows that the rate of detected $M$. pneumoniae infections was overall low from 2006 to 2009 (between $2 \%$ and $7 \%$, with seasonal peaks between June and August. However, in December 2009 the rate of $M$. pneumoniae infections quickly rose to $21 \%(15 / 70)$ and remained high through to November 2011, when $11 \%(13 / 115)$ of patients with RTIs tested M. pneumoniae positive. Only later did the rate of $M$. pneumoniae infections return below the 2006 to 2014 average (9\%) and remained low, with seasonal peaks during the summer, similarly to the time before 2010. Another unusual increase in the rate of $M$. pneumoniae positive samples occurred after the typical summer peak in 2013, during winter 2013 and spring 2014, until November 2014, when $37 \%$ (214/580) of patients were positive by M. pneumoniae PCR (Figure). It was also found that $15 \%(436 / 2,996)$ of patients in Ljubljana and $13 \%(819 / 6,435)$ in other parts of the Central Slovenian region had $M$. pneumoniae acute infections, which seems to indicate that $M$. pneumoniae was overall similarly present in the geographical area included in the study. Additionally, the rise in the number of $M$. pneumoniae positive patients during 2010 and 2014 occurred almost evenly throughout the whole studied area. We also did not observe 


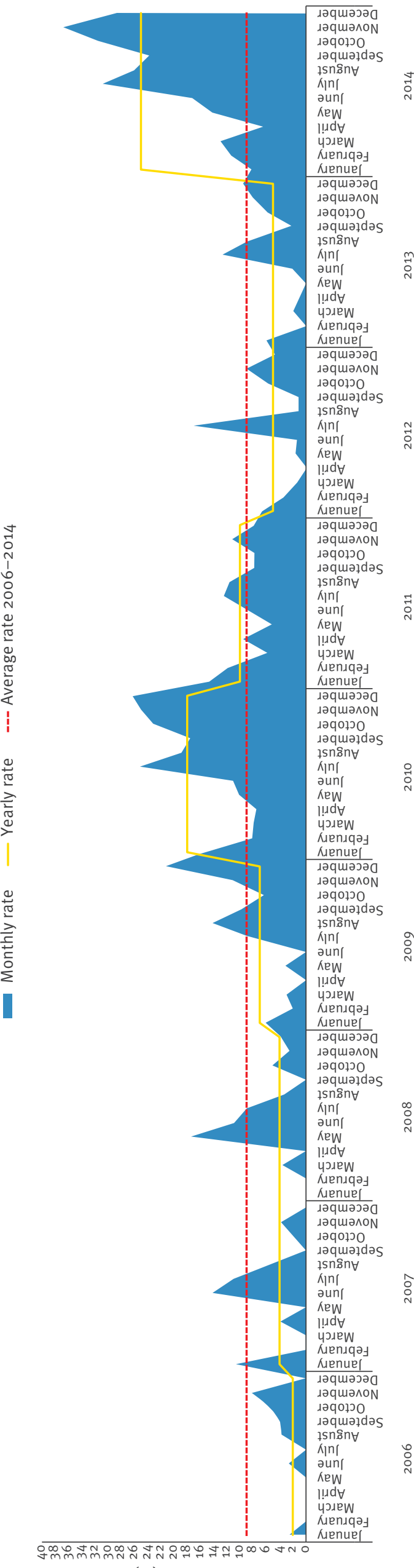

(\%) әреу any specific clustering that could indicate a merely localised outbreak during the time of the study.

Of the 1,255 PCR positive samples, M. pneumoniae was successfully cultured from $62 \%(n=783)$. The distribution of the obtained isolates according to sex was $50 \%$ (390/783) men, 50\% (393/783) women, and overall $78 \%(614 / 783)$ of cultured samples were from children and $22 \%(169 / 783)$ from adults.

\section{Mycoplasma pneumoniae genotyping and} macrolide resistance detection

For all 783 M. pneumoniae cultured isolates, PCR products were successfully obtained for all targets, namely MPN141, MPN528a and both parts of 23S rRNA gene domain $\mathrm{V}$ (Assay 1 and Assay 2). All assays produced good quality pyrosequencing products of $7,10,79$ and $16 \mathrm{nt}$ for MPN141, MPN528a, 23S rRNA domain V Assay 1 and Assay 2, respectively. During the assessed time period, $M$. pneumoniae $\mathrm{P}_{1}$ type I and $\mathrm{P}_{1}$ type II strains were found in $60 \%(469 / 783)$ and $40 \%(312 / 783)$ of cultured isolates, respectively. We also detected two isolates with both $\mathrm{P}_{1}$ type I and $\mathrm{P}_{1}$ type II M. pneumoniae DNA.

A more detailed analysis on a year-to-year basis showed that although both $\mathrm{P}_{1}$ types remained present during the whole study period, the most observed $M$. pneumoniae $\mathrm{P}_{1}$ type during the 2010 epidemic was $\mathrm{P}_{1}$ type II $\left(82 \%\right.$; 81/99), while in the 2014 epidemic $P_{1}$ type I dominated $(75 \%$; 384/510) (Table 2). Moreover it seems that between these two epidemic years, the proportion of isolates with $\mathrm{P}_{1}$ type II decreased in favour of $\mathrm{P}_{1}$ type I (Table 2).

Seven samples ( $1 \% ; 7 / 783)$, all from inpatients, were found to contain an A2063G mutation (Table 2). Six mutated strains were cultured from respiratory samples of paediatric patients and one from an adult patient. Three of the presumed macrolide resistant strains belonged to the $\mathrm{P}_{1}$ type I and four to the $\mathrm{P}_{1}$ type II. All other isolated $M$. pneumoniae strains had no mutation in the two sequenced areas of the 23S rRNA gene and were therefore presumed to be macrolide sensitive.

\section{Mycoplasma pneumoniae 23S rRNA} polymerase chain reaction Assay 1 and Assay 2 specificity

As predicted by the NCBI BLAST search, no tested organism except $M$. genitalium produced a positive PCR result in either M. pneumoniae 23 S rRNA Assay 1 or Assay 2. However, after pyrosequencing the PCR product from Assay 1, the sequence of $M$. pneumoniae had six differences (73/79; $92 \%$ identity) distinguishing it from M. genitalium.

\section{Discussion}

From our data, we observed that in 2006, 2007, 2008, 2009, 2012 and 2013 the number of M. pneumoniae positive cases usually rose during the summer but quickly returned to low values during the autumn, 


\section{TABLE 2}

Annual number of Mycoplasma pneumoniae isolates, P1 type distribution, percentage of inferred macrolideresistant strains and detected $23 \mathrm{~S}$ rRNA mutations, Central Slovenian region, 2006-2014

\begin{tabular}{|c|c|c|c|c|c|c|}
\hline Year & $\begin{array}{l}\text { Number } \\
\text { of } \\
\text { isolates }\end{array}$ & $\begin{array}{c}\text { P1 type I } \\
N(\%)\end{array}$ & $\begin{array}{c}\text { P1 type II } \\
\text { N (\%) }\end{array}$ & $\begin{array}{c}\text { P1 type } \\
\text { I+ II } \\
N(\%)\end{array}$ & $\begin{array}{l}\text { Mutated } \\
\text { strains N } \\
\quad(\%)\end{array}$ & $\begin{array}{l}23 \mathrm{~S} \text { rRNA } \\
\text { mutation }\end{array}$ \\
\hline 2006 & 4 & $2(50)$ & $2(50)$ & o (o) & o (o) & None \\
\hline 2007 & 19 & $9(47)$ & $10(53)$ & $\mathrm{o}(\mathrm{o})$ & o (o) & None \\
\hline 2008 & 7 & $2(29)$ & $5(71)$ & o (o) & o (o) & None \\
\hline 2009 & 33 & $12(36)$ & $21(64)$ & o (o) & $1(3)$ & A2063G \\
\hline 2010 & 99 & $17(17)$ & $81(82)$ & $1(1)$ & $2(2)$ & A2063G \\
\hline 2011 & 46 & $12(26)$ & $33(72)$ & $1(2)$ & o (o) & None \\
\hline 2012 & 29 & $8(28)$ & $21(72)$ & $\mathrm{o}(\mathrm{o})$ & $\mathrm{o}(0)$ & None \\
\hline 2013 & 36 & $23(64)$ & $13(36)$ & $\mathrm{o}(\mathrm{o})$ & $1(3)$ & A2063G \\
\hline 2014 & 510 & $384(75)$ & $126(25)$ & $\mathrm{o}(\mathrm{o})$ & $3(1)$ & A2063G \\
\hline Total & 783 & $469(60)$ & $312(40)$ & $2(0)$ & $7(1)$ & - \\
\hline
\end{tabular}

winter and spring, suggesting that in these years the occurrence of $M$. pneumoniae infections was most likely endemic. On the other hand, a different pattern with unusual increases in $M$. pneumoniae infections was seen from November 2009 to December 2011 and from May 2014 to December 2014. Based on these results, we suggest that a $M$. pneumoniae epidemic took place in Slovenia from November 2009 to December 2011 similarly to other European countries [42]. Interestingly, our results also seem to show that another, even more extensive, outbreak of $M$. pneumoniae infections began in May 2014, reached its peak in November 2014 , when $37 \%$ (214/580) of patients were positive for M. pneumoniae DNA and persisted at least until December 2014, when 29\% (171/598) of patients were infected. Comparable results from other countries are not yet available, so we cannot say whether such a scenario is limited to Slovenia or correlates with a new European-wide epidemic.

We do not believe that the rises in the number of $M$. pneumoniae positive patients were due to increased awareness or testing availability, since no changes were made in the healthcare system in Slovenia during the time of the study. Additionally, the same test was used throughout and increased numbers of positives cannot therefore be ascribed to methodological changes in sensitivity. Moreover, a similar increase in the number of $M$. pneumoniae cases was also observed by other laboratories in Slovenia (data not shown). Finally, the number of tested patients did increase from 2006 to 2014 but most likely as a result of more patients seeking help at healthcare facilities during epidemics.

Analysis of $M$. pneumoniae $\mathrm{P}_{1}$ type distribution in Central Slovenia from 2006 to 2014 shows that both $\mathrm{P}_{1}$ types were present in the studied population during the whole period. No complete type shift phenomenon, as described in a Japanese study [43], could be observed in our population but it would seem that the
2010/11 epidemic was caused mostly by $M$. pneumoniae $\mathrm{P}_{1}$ type II and the 2014 epidemic by $M$. pneumoniae $\mathrm{P}_{1}$ type I. Although we observed a large increase in $M$. pneumoniae cases in 2014, we cannot be sure that the reason for the new outbreak was an increase in the number of $P_{1}$ type I strains. Further studies using multilocus variable-number tandem repeat analysis (MLVA) [44] are needed to assess whether the 2010/11 and 2014 epidemics were caused by a specific MLVA type or multiple types, as seen in a recent study from France and Israel [45]. On the basis of $\mathrm{P}_{1}$ typing, however, it might be concluded that the prevalence of the two major $M$. pneumoniae genetic types in Slovenia does oscillate during time.

The results of our study suggest that macrolide-resistant $M$. pneumoniae strains, although represented by small numbers of isolates, are already present in Slovenian patients, which seems to be in concordance with most European studies, which also show low level of macrolide resistance in $M$. pneumoniae $[11,14,15,17]$. An exception is a study from 2011 , in which macrolide resistance was detected in $26 \%$ of children presenting with $M$. pneumoniae pneumonia or bronchitis in an Italian city [16].

Our low prevalence of $M$. pneumoniae macrolide resistant strains, however, is not unexpected, since a national antibiotic usage survey shows that macrolides are being carefully used in Slovenia with Daily Doses (DD) per 1,000 inhabitants of $2.14,2.43,2.22,2.11$, $1.85,1.75,1.84,1.67$ and 1.65 in 2006, 2007, 2008, 2009, 2010, 2011, 2012, 2013 and 2014, respectively $[46,47]$. Interestingly, M. pneumoniae macrolide-resistant strains were first detected in 2009, and then in 2010, 2013 and 2014, when macrolide antibiotic DDs per 1,000 inhabitants were constantly declining.

We noted that all mutated strains harbour an A2063G substitution, which is recognised as the most prevalent and high level macrolide resistance mediating mutation [27]. Although all strains harboured the same type of mutation, we did not find any connection among the patients from whom these were derived. The patients were from different geographical parts of Central Slovenia and the dates of sample collection were several months or even years apart. Moreover, basic molecular typing studies showed that three presumed macrolide-resistant strains belonged to $\mathrm{P}_{1}$ type I and four to $\mathrm{P}_{1}$ type II subgroup, which additionally supports a lack of connection between the strains. Studies using more accurate genetic methods (MLVA) could shed further light on the phylogeny of these strains.

To summarise, we observed two epidemics of $M$. pneumoniae infections. The first was, similarly to some other European countries, in 2010/11. The second epidemic seems to have started in Slovenia in 2014. We also found that $M$. pneumoniae $\mathrm{P}_{1}$ type II may have been the main cause of the 2010 epidemic while $P_{1}$ type I may have been responsible for the new 2014 
outbreak. Additionally, in concordance with the situation in Europe, we documented the presence of macrolide-resistant $M$. pneumoniae strains in children and in adults at very low level.

\section{Acknowledgements}

The authors would like to express their gratitude to Mateja Jovanović, Ines Bernjak-Šimaga and Sabina Pervić for their technical help. All the work has been funded by the University of Ljubljana, Medical Faculty, Institute of Microbiology and Immunology.

\section{Conflict of interest}

None declared.

\section{Authors' contributions}

RK: execution of experiments, analysis and interpretation of results, manuscript writing; DK: project leader, analysis and interpretation of results, manuscript writing, TM and MP: sample collection, patient data, pre-submission manuscript reviewing.

\section{References}

1. HammerschlagMR. Mycoplasma pneumoniae infections.Cur Opin Infect Dis. 2001;14(2):181-6. DOI: 10.1097/00001432200104000-00012 PMID: 11979130

2. AtkinsonTP, BalishMF, WaitesKB. Epidemiology, clinical manifestations, pathogenesis and laboratory detection of Mycoplasma pneumoniae infections.FEMS Microbiol Rev. 2008;32(6):956-73. DOI: 10.1111/j.1574-6976.2008.00129.x PMID: 18754792

3. McIntoshK. Community-acquired pneumonia in children.N Engl J Med. 2002;346(6):429-37. DOI: 10.1056/NEJMra011994 PMID: 11832532

4. DefilippiA, SilvestriM, TacchellaA, GiacchinoR, MelioliG, Di MarcoE, et al. Epidemiology and clinical features of Mycoplasma pneumoniae infection in children. Respir Med. 2008;102(12):1762-8. DOI: 10.1016/j.rmed.2008.06.022 PMID: 18703327

5. HowardLS, SillisM, PasteurMC, KamathAV, HarrisonBD. Microbiological profile of community-acquired pneumonia in adults over the last 20 years.J Infect. 2005;50(2):107-13. DOI: 10.1016/j.jinf.2004.05.003 PMID: 15667910

6. MiyashitaN, OuchiK, KawasakiK, OdaK, KawaiY, ShimizuH, et al. Mycoplasma pneumoniae pneumonia in the elderly. Med Sci Monit. 2008;14(8):CR387-91.PMID: 18667994

7. LiX, AtkinsonTP, HagoodJ, MakrisC, DuffyLB, WaitesKB. Emerging macrolide resistance in Mycoplasma pneumoniae in children: detection and characterization of resistant isolates.Pediatr Infect Dis J. 2009;28(8):693-6. DOI: 10.1097/ INF.obo13e31819e3f7a PMID: 19633515

8. RasmussenJN, VoldstedlundM, AndersenRL, EllermannEriksenS, JensenTG, JohansenHK, et al. Increased incidence of Mycoplasma pneumoniae infections detected by laboratorybased surveillance in Denmark in 2010. Euro Surveill. 2010;15(45):19708.PMID: 21087593

9. ChalkerV, StockiT, MentastiM, FlemingD, HarrisonT. Increased incidence of Mycoplasma pneumoniae infection in England and Wales in 2010: multiocus variable number tandem repea analysis typing and macrolide susceptibility.Euro Surveill. 2011;16(19):19865.PMID: 21596009

10. EunBW, KimNH, ChoiEH, LeeHJ. Mycoplasma pneumoniae in Korean children: the epidemiology of pneumonia over an 18-year period.J Infect. 2008;56(5):326-31. DOI: 10.1016/j. jinf.2008.02.018 PMID: 18420275

11. PereyreS, CharronA, RenaudinH, BébéarC, BébéarCM. First report of macrolide-resistant strains and description of a novel nucleotide sequence variation in the $P_{1}$ adhesin gene in Mycoplasma pneumoniae clinical strains isolated in France over 12 years.J Clin Microbiol. 2007;45(11):3534-9. DOI: 10.1128/JCM.01345-07 PMID: 17881549
12. Cousin A, Bertille DB, Alain C, Helene R, Christiane B. Analysis of RFLPs amplified cytadhesin P1 gene for epidemiological study of Mycoplasma pneumoniae. In: Programme and abstracts of the 1oth International Congress of the International Organisation for Mycoplasmology (IOM). 1994; p. 494-5.

13. Dorigo-ZetsmaJW, DankertJ, ZaatSA. Genotyping of Mycoplasma pneumoniae clinical isolates reveals eight P1 subtypes within two genomic groups.J Clin Microbiol. 2000;38(3):965-70.PMID: 10698981

14. UldumSA, BangsborgJM, Gahrn-HansenB, LjungR, MølvadgaardM, Føns PetersenR, et al. Epidemic of Mycoplasma pneumoniae infection in Denmark, 2010 and 2011. Euro Surveill. 2012;17(5):20073.PMID: 22321137

15. DumkeR, von BaumH, LückPC, JacobsE. Occurrence of macrolide-resistant Mycoplasma pneumoniae strains in Germany.Clin Microbiol Infect. 2010;16(6):613-6. DOI: 10.1111/j.1469-0691.2009.02968.x PMID: 19765022

16. ChironnaM, SallustioA, EspositoS, PerulliM, Chinellatol, Di BariC, et al. Emergence of macrolide-resistant strains during an outbreak of Mycoplasma pneumoniae infections in children. J Antimicrob Chemother. 2011;66(4):734-7. DOI: 10.1093/jac/ dkroo3 PMID: 21393214

17. PeuchantO, MénardA, RenaudinH, MorozumiM, UbukataK, BébéarCM, et al. Increased macrolide resistance of Mycoplasma pneumoniae in France directly detected in clinical specimens by real-time PCR and melting curve analysis. J Antimicrob Chemother. 2009;64(1):52-8. DOI: 10.1093/jac/ dkp16o PMID: 19429926

18. AverbuchD, Hidalgo-GrassC, MosesAE, EngelhardD, Nir-PazR. Macrolide resistance in Mycoplasma pneumoniae, Israel, 2010.Emerg Infect Dis. 2011;17(6):1079-82. DOI: 10.3201/ eid/1706.101558 PMID: 21749775

19. YamadaM, BullerR, BledsoeS, StorchGA. Rising rates of macrolide-resistant Mycoplasma pneumoniae in the central United States.Pediatr Infect Dis J. 2012;31(4):409-10. DOI: 10.1097/INF.obo13e318247f3eo PMID: 22209916

20. Acute Respiratory Diseases Study Group,MorozumiM, IwataS, HasegawaK, ChibaN, TakayanagiR, MatsubaraK, et al. Increased macrolide resistance of Mycoplasma pneumoniae in pediatric patients with community-acquired pneumonia. Antimicrob Agents Chemother. 2008;52(1):348-50. DOI: 10.1128/AAC.00779-07 PMID: 17954691

21. CaoB, ZhaoCl, YinYD, ZhaoF, SongSF, Bail, et al. High prevalence of macrolide resistance in Mycoplasma pneumoniae isolates from adult and adolescent patients with respiratory tract infection in China. Clin Infect Dis. 2010;51(2):189-94. DOI: 10.1086/653535 PMID: 20540621

22. XinD, MiZ, HanX, QinL, LiJ, WeiT, et al. Molecular mechanisms of macrolide resistance in clinical isolates of Mycoplasma pneumoniae from China. Antimicrob Agents Chemother. 2009;53(5):2158-9. DOI: 10.1128/AAC.01563-08 PMID: 19273685

23. LiuY, YeX, ZhangH, XuX, LiW, ZhuD, et al. Antimicrobial susceptibility of Mycoplasma pneumoniae isolates and molecular analysis of macrolide-resistant strains from Shanghai, China. Antimicrob Agents Chemother. 2009;53(5):2160-2. DOI: 10.1128/AAC.01684-08 PMID: 19273684

24. ZhaoF, LvM, TaoX, HuangH, ZhangB, ZhangZ, et al. Antibiotic sensitivity of 40 Mycoplasma pneumoniae isolates and molecular analysis of macrolide-resistant isolates from Beijing, China. Antimicrob Agents Chemother. 2012;56(2):1108-9. DOI: 10.1128/AAC.05627-11 PMID: 22106216

25. KawaiY, MiyashitaN, YamaguchiT, SaitohA, KondohE, FujimotoH, et al. Clinical efficacy of macrolide antibiotics against genetically determined macrolide-resistant Mycoplasma pneumoniae pneumonia in paediatric patients. Respirology. 2012;17(2):354-62. DOI: 10.1111/j.14401843.2011.02102.x PMID: 22077195

26. BébéarCM, PereyreS. Mechanisms of drug resistance in Mycoplasma pneumoniae.Curr Drug Targets Infect Disord. 2005;5(3):263-71. DOI: 10.2174/1568005054880109 PMID: 16181145

27. MorozumiM, TakahashiT, UbukataK. Macrolide-resistant Mycoplasma pneumoniae: characteristics of isolates and clinical aspects of community-acquired pneumonia.J Infect Chemother. 2010;16(2):78-86. DOI: 10.1007/s10156-009-0021-4 PMID: 2009475

28. LiuY, YeX, ZhangH, XuX, LiW, ZhuD, et al. Characterization of macrolide resistance in Mycoplasma pneumoniae isolated from children in Shanghai, China. Diagn Microbiol Infect Dis. 2010;67(4):355-8. DOI: 10.1016/j.diagmicrobio.2010.03.004 PMID: 20638604

29. SocanM, Marinic-FiserN, KraigherA, KotnikA, LogarM. Microbial aetiology of community-acquired pneumonia 
in hospitalised patients. Eur J Clin Microbiol Infect Dis. 1999;18(11):777-82. DOI: $10.1007 /$ S100960050400 PMID: 10614951

30. BeovićB, BonacB, KeseD, Avsic-ZupancT, KreftS, LesnicarG, et al. Aetiology and clinical presentation of mild communityacquired bacterial pneumonia. Eur J Clin Microbiol Infect Dis. 2003;22(10):584-91. DOI: 10.1007/S10096-003-0997-0 PMID: 13680399

31. Population, statistical regions, Slovenia, annualy. Republic of Slovenia Statistical office RS. [Accessed 24 Jun 2015]. Available from: http://pxweb.stat.si/pxweb/Dialog/varval. asp $? \mathrm{ma}=05 \mathrm{C} 2002 \mathrm{E} \& \mathrm{ti}=\&$ path $=. . /$ Database $/$ Demographics $/ 05$ population/10_Number_Population/10_05C20_Population_ stat_regije/\&lang=1

32. Waites KB, Rikihisa Y, Taylor-Robinson D. Mycoplasma and Ureaplasma. In: Murray PR, Baron EJ, Jorgensen JH, Pfaller MA, Yolken RH, editors. Manual of clinical microbiology 8th edition. Washington DC: American Society for Microbiology Press; 2003. p. 972-90.

33. PereyreS, GuyotC, RenaudinH, CharronA, BébéarC, BébéarCM. In vitro selection and characterization of resistance to macrolides and related antibiotics in Mycoplasma pneumoniae. Antimicrob Agents Chemother. 2004;48(2):460-5. DOI: 10.1128/AAC.48.2.460-465.2004 PMID: 14742195

34. HimmelreichR, HilbertH, PlagensH, PirklE, LiBC, HerrmannR. Complete sequence analysis of the genome of the bacterium Mycoplasma pneumoniae. Nucleic Acids Res. 1996;24(22):442049. DOI: $10.1093 / \mathrm{nar} / 24.22 .4420$ PMID: 8948633

35. SchwartzSB, MitchellSL, ThurmanKA, WolffBJ, WinchellJM. Identification of $P_{1}$ variants of Mycoplasma pneumoniae by use of high-resolution melt analysis.J Clin Microbiol. 2009;47(12):4117-20. DOI: 10.1128/JCM.01696-09 PMID: 19828737

36. LinC, LiS, SunH, ZhaoH, FengY, CaoL, et al. Nested PCR-linked capillary electrophoresis and single-strand conformation polymorphisms for detection of macrolide-resistant Mycoplasma pneumoniae in Beijing, China. J Clin Microbiol. 2010;48(12):4567-72. DOI: 10.1128/JCM.00400-10 PMID: 20861333

37. SasakiT, KenriT, OkazakiN, IsekiM, YamashitaR, ShintaniM, et al. Epidemiological study of Mycoplasma pneumoniae infections in japan based on PCR-restriction fragment length polymorphism of the $\mathrm{P}_{1}$ cytadhesin gene. J Clin Microbiol. 1996;34(2):447-9.PMID: 8789036

38. MatsuokaM, NaritaM, OkazakiN, OhyaH, YamazakiT, OuchiK, et al. Characterization and molecular analysis of macrolideresistant Mycoplasma pneumoniae clinical isolates obtained in Japan. Antimicrob Agents Chemother. 2004;48(12):4624-30. DOI: $10.1128 / A A C .48 .12 .4624-4630.2004$ PMID: 15561835

39. ZhaoF, CaoB, LiJ, SongS, TaoX, YinY, et al. Sequence analysis of the $\mathrm{p} 1$ adhesin gene of Mycoplasma pneumoniae in clinical isolates collected in Beijing in 2008 to 2009. J Clin Microbiol. 2011;49(8):3000-3. DOI: 10.1128/JCM.00105-11 PMID: 21697320

40. SpuesensEB, HoogenboezemT, SluijterM, HartwigNG, van RossumAM, VinkC. Macrolide resistance determination and molecular typing of Mycoplasma pneumoniae by pyrosequencing.J Microbiol Methods. 2010;82(3):214-22. DOI: 10.1016/j.mimet.2010.06.004 PMID: 20547188

41. SpuesensEB, MeijerA, BierschenkD, HoogenboezemT, DonkerGA, HartwigNG, et al. Macrolide resistance determination and molecular typing of Mycoplasma pneumoniae in respiratory specimens collected between 1997 and 2008 in The Netherlands. J Clin Microbiol. 2012;50(6):1999-2004. DOI: 10.1128/JCM.00400-12 PMID: 22495561

42. European Working Group on Mycoplasma pneumoniae surveillance, LengletA, HerradorZ, MagiorakosAP, LeitmeyerK, CoulombierD. Surveillance status and recent data for Mycoplasma pneumoniae infections in the European Union and European Economic Area, January 2012. Euro Surveill. 2012;17(5):20075.PMID: 22321134

43. KenriT, OkazakiN, YamazakiT, NaritaM, IzumikawaK, MatsuokaM, et al. Genotyping analysis of Mycoplasma pneumoniae clinical strains in Japan between 1995 and 2005: type shift phenomenon of $M$. pneumoniae clinical strains. J Med Microbiol. 2008;57(Pt 4):469-75. DOI: 10.1099/ jmm.0.47634-o PMID: 18349367

44. DiazMH, BenitezAJ, WinchellJM. Investigations of Mycoplasma pneumoniae infections in the United States: trends in molecular typing and macrolide resistance from 2006 to 2013.J Clin Microbiol. 2015;53(1):124-30. DOI: $10.1128 / J C M .02597-14$ PMID: 25355769

45. PereyreS, CharronA, Hidalgo-GrassC, TouatiA, MosesAE, Nir-PazR, et al. The spread of Mycoplasma pneumoniae is polyclonal in both an endemic setting in France and in an epidemic setting in Israel. PLoS ONE. 2012;7(6):e38585. DOI: 10.1371/journal.pone.0038585 PMID: 22701675

46. Pečar-Čad S, Hribovšek T. 2012. Ambulantno predpisovanje zdravil v sloveniji po ACT klasifikaciji v letu 2011. [Outpatient prescription drugs in Slovenia according to The Anatomical Therapeutic Chemical (ATC) Classification System in 2011]. Ljubljana: Inštitut za varovanje zdravja republike Slovenije. [Accessed 7 Oct 2014]. Slovenian. Available from: http:// www.nijz.si/sites/www.nijz.si/files/publikacije-datoteke/ ambulantno_predpisovanje_zdravil_v_slo_po_atc_ klasifikaciji_2011.pdf

47. The European Surveillance System (TESSy) - Distribution of antimicrobial consumption by antimicrobial group. Stockholm: European Centre for Disease Prevention and Control. [Accessed 22 Jun 2015]. Available from: http://www.ecdc.europa.eu/en/ healthtopics/antimicrobial resistance/esac-net-database/ Pages/consumption-rates-by-country.aspx 